\title{
Seven-year follow-up of posterior chamber phakic intraocular lens with central port design
}

\author{
Luis Fernández-Vega-Cueto ${ }^{1}$, Belén Alfonso-Bartolozzi ${ }^{1}$, Carlos Lisa ${ }^{1}$, David Madrid-Costa ${ }^{2}$ and José F. Alfonso ${ }^{1 *}$ (D)
}

\begin{abstract}
Background: To assess the clinical outcomes of the Visian Implantable Collamer Lens (ICL) with a central port throughout 7 years of follow-up.

Methods: Eighty-four eyes of 52 patients were evaluated over a follow-up period of 7 years after V4C ICL implantation. Uncorrected (UDVA) and corrected (CDVA) distance visual acuities, refraction, intraocular pressure $(\mathrm{IOP})$, endothelial cell density (ECD) and vault were analysed.

Results: The mean postoperative UDVA (logMAR) was $0.04 \pm 0.11,0.13 \pm 0.19$ and $0.17 \pm 0.23$ at 1-, 5- and 7-years, respectively $(P<0.0001)$. The mean CDVA (logMAR) remains unchanged throughout a 7-year follow-up period $(0.02 \pm 0.08$ and $0.02 \pm 0.08$, at 5 - and 7-years, respectively, $P=0.2)$. At all follow-up visits, more than $95 \%$ of the eyes achieved a CDVA of 20/25 or better and more than $85 \%$ a CDVA of 20/20. At the end of the follow-up (7 years), no eye lost more lines of CDVA, 56 eyes (66.7\%) and 28 eyes (33.3\%) gained lines of CDVA. At 7-years, the spherical equivalent was $-0.62 \pm 0.62 \mathrm{D}$. No significant increase in IOP (> $20 \mathrm{mmHg}$ or an increase higher than $5 \mathrm{mmHg}$ ) occurred in any case throughout the 7-year of follow-up. The loss in ECD from the preoperative baseline at the last follow-up visit was $2.6 \%$. No intraoperative or postoperative complications or adverse events occurred during the follow-up period.
\end{abstract}

Conclusions: The outcomes of this study show the long-term viability of the $\mathrm{V} 4 \mathrm{C} \mathrm{ICL}$ implantation as a surgical option for the correction of myopia.

Keywords: Implantable Collamer Lens, ICL, Myopia, Long-term

\section{Background}

The Visian Implantable Collamer Lens (ICL) (Staar Surgical AG, Nidau, Switzerland) with a central port design (V4c), has been widely accepted as effective and safe treatment for myopia correction [1]. The central port design (named KS-Aquaport; as a tribute to his inventor; Kimiya Shimizu) allows the circulation of the aqueous fluid through the lens [2,3]. The lens was implanted first

\footnotetext{
* Correspondence: j.alfonso@fernandez-vega.com

${ }^{1}$ Fernández-Vega Ophthalmological Institute, Avda. Dres. Fernández-Vega 114, 33012 Oviedo, Spain

Full list of author information is available at the end of the article
}

in 2007, since then, several studies with small and large samples have shown their results at different follow-ups supporting the use of this lens for the correction of moderate to high myopia [1].

Compared to the previous ICL models, the incidence of reported complications has significantly decreased with the V4c model $[1,4]$. For example, the reported incidence of cataracts, which is the most common and significant complication with the previous model [4], has been reduced to almost 0 with the V4c model $[1,5]$, even for those cases with a low vault [6]. These findings support the idea that aqueous humour flow through the 
central hole of the lens maintains the normal physiology of the anterior segment of the eye, preventing potential complications. However, it should be noted there have been only a few long-term studies (spanning more than 3 years) [6-11] on the clinical outcomes of the V4c ICL implantation, and only three studies reached 5-years follow-up [6-8]. In contrast, several of the long-term studies (spanning more than 5 years) of the previous ICL design have been published [12-19]. Cumulative numbers of complications of ICL implantation are expected to increase with time $[12,16,17,20]$. Furthermore, Yang et al. [11] recently, in a 4-year prospective study, found that the average lens density increased at 4 years after ICL V4c implantation, although no cataracts were reported during follow-up. It should be confirmed whether, over a longer follow-up, those changes in lens density will lead to a clinically significant cataract.

Although the ICL V4c implantation has shown to provide outstanding and stable visual and refractive outcomes and low adverse event rates, the follow-up periods in most of the studies ranged between 1 to 3 years [1], and there is a paucity of long-term studies of this new ICL model. This study aims to evaluate longterm clinical outcomes of the ICL V4c implantation for moderate to high myopia throughout a 7-year follow-up period.

\section{Methods}

This retrospective, observational study comprised 84 eyes of 52 patients who underwent implantation of the Visian Implantable Collamer Lens (ICL, model V4c, STAAR Surgical Inc) to correct myopia at the Fernández-Vega Ophthalmological Institute, Oviedo, Spain, from January to December 2012. All patients provided written informed consent after the nature and possible consequences of the study were explained fully in accordance with the Declaration of Helsinki. The inclusion criteria were stable refraction with a myopic error in the range correctable with the V4c ICL (from - $1.00 \mathrm{D}$ to - 18.00 D of sphere), a clear central cornea, anterior chamber depth (ACD) greater than $2.8 \mathrm{~mm}$ measured from the corneal endothelium to the anterior lens capsule, endothelial cell density (ECD) greater than 2000 cells $/ \mathrm{mm}^{2}$, mesopic pupil smaller than $7.0 \mathrm{~mm}$, trabecular-iris angle (TIA) greater than $35^{\circ}$ (grade III by gonioscopy), crystalline lens rise (CLD) less than $500 \mu \mathrm{m}$ and postoperative follow-up period of at least 7 years. The exclusion criteria were cataract, history of glaucoma or retinal detachment, macular degeneration or retinopathy, neuro-ophthalmic disease, or any ocular inflammation history.

Before the surgery, patients had a complete ophthalmologic examination, including uncorrected distance visual acuity (UDVA), corrected distance visual acuity
(CDVA), manifest and cycloplegic refractions, slit lamp examination, keratometry, corneal topography, pachymetry and white-to-white (Sirius, CSO Ophthalmic, Italy), ACD and angle to angle (OCT Visante, Carl Zeiss Meditec, Germany \&), ECD measurement (SP 3000P, Topcon Europe Medical), intraocular pressure (IOP) measurement by Goldmann applanation tonometry, and anterior segment optical coherence tomography (OCT; Visante, Carl Zeiss Meditec AG).

All included eyes in this study had implantation of the myopic V4c Visian ICL model. The details of the lens have been published previously [8]. Emmetropia was selected as the postoperative target refraction for all eyes. ICL power calculation was performed using a modified vertex formula provided by the manufacturer (Staar Surgical). ICL size was individually determined based on the horizontal white-to-white (WTW) distance, ACD measured by Scheimpflug photography, and angle-to-angle distance measured with OCT. To prevent a postoperative vault greater than $1000 \mu \mathrm{m}$, the following protocol, based on the surgeon's experience, was applied: In cases where the distance from the ICL to the angle-to-angle diameter (ATA) was higher than $800 \mu \mathrm{m}$, or had a pupillary ovalization or compromised pupillary dynamic in the postoperative visit of the first day, we verified that the vertical angle-angle was longer than the horizontal, and subsequently, the ICL was rotated $90^{\circ}$ to vertical orientation.

The same experienced surgeon (JFA) performed all surgeries following the standard procedure previously described $[8,9,21]$. Postoperative follow-up visits were scheduled at 1-day, 1 week and at 1, 3 and 12 months and then every 1 year thereafter. The analysis included the outcomes from preoperative, and 1-, 5-, and 7-years visits. The examinations included measurement of UDVA and CDVA, manifest refraction, slit-lamp examination, IOP, ECD and fundoscopy. The central distance between the ICL and the crystalline lens (vault) was assessed using OCT. The vault between the crystalline lens and the ICL was measured perpendicular to the lens apex or at the narrowest point.

Data analysis was performed using SPSS for Windows, version 14.0 (SPSS Inc., Chicago, IL). Normality was checked with the Kolmogorov-Smirnov test. One-way repeated-measures analysis of variance (ANOVA) with a Bonferroni post-hoc test was performed to compare results. Differences were considered to be statistically significant when the $P$ value was less than 0.05 .

\section{Results}

This study included 84 eyes of 52 patients (17 men and 35 women). All patients completed the follow-up period of 7 years and attended all the follow-up visits. Table 1 summarizes preoperative demographic data of 
Table 1 Preoperative patient demographics and ICL characteristics

\begin{tabular}{lll}
\hline & Mean \pm SD & Range [Min, Max] \\
\hline Age (years) & $31.04 \pm 4.89$ & {$[25,50]$} \\
Refraction sphere (D) & $-9.02 \pm 2.85$ & {$[-17.50,-4.0]$} \\
Refraction cylinder (D) & $-0.65 \pm 0.51$ & {$[-1.5,0]$} \\
Spherical Equivalent (D) & $-9.35 \pm 2.90 .60$ & {$[-18.25,-4.50]$} \\
UDVA (logMAR) & $1.63 \pm 0.38$ & {$[0.7,2.0]$} \\
CDVA (logMAR) & $0.04 \pm 0.12$ & {$[0.0,0.4]$} \\
Minimum Keratometry (D) & $43.26 \pm 1.64$ & {$[40.00,46.00]$} \\
Maximum Keratometry (D) & $44.18 \pm 1.69$ & {$[40.78,47.00]$} \\
Corneal thickness ( $\mu$ m) & $530 \pm 37$ & {$[448,630]$} \\
ACD (mm) & $3.13 \pm 0.22$ & {$[2.80,3.75]$} \\
WTW (mm) & $11.95 \pm 0.47$ & {$[10.99,13.45]$} \\
ATA (mm) & $11.74 \pm 0.37$ & {$[11.00,13.20]$} \\
ECD (cells/ mm ${ }^{2}$ ) & $2640 \pm 336$ & {$[2000,3903]$} \\
IOP (mmHg) & $13.02 \pm 1.78$ & {$[8,19]$} \\
ICL sphere (D) & $-10.17 \pm 2.70$ & {$[-18.0,-5.0]$} \\
ICL size (mm) & $13.10 \pm 0.33$ & {$[12.6,13.7]$} \\
\hline
\end{tabular}

ICL implantable collamer lens, $D$ dioptres, UDVA uncorrected distance visual acuity, CDVA corrected distance visual acuity, $A C D$ anterior chamber depth, WTW white to white, ATA angle to angle, ECD endothelial cell density, IOP intraocular pressure, $S D$ standard deviation

the patients and ICL characteristics. The distribution of the lens sizes implanted were: $13.7 \mathrm{~mm}$ in 9 eyes (10.7\%), $13.2 \mathrm{~mm}$ in 53 eyes (63.1\%), and $12.6 \mathrm{~mm}$ in 22 eyes (26.2\%).

\section{Effectiveness and safety outcomes}

The mean postoperative UDVA (logMAR) was $0.04 \pm$ $0.11,0.13 \pm 0.19$ and $0.17 \pm 0.23$ at 1 -, 5 - and 7-years, respectively $(P<0.0001)$. The efficacy index (mean postoperative UDVA/mean preoperative CDVA) was 1.01, 0.85 and 0.80 at 1-, 5- and 7-years after surgery, respectively. Figure 1a shows the cumulative UDVA at each follow-up visit. The mean CDVA increased from the preoperative $0.04 \pm 0.12 \log$ MAR to $0.01 \pm 0.06 \log$ MAR at 1 -year after surgery $(P<0.0001)$ and remained unchanged throughout a 7-year follow-up period $(0.02 \pm$ $0.08 \log$ MAR and $0.02 \pm 0.08 \log M A R$, at 5 - and 7-years, respectively, $P=0.2$ ). At all follow-up visits, more than $95 \%$ of the eyes achieved a CDVA of 20/25 or better and more than $85 \%$ a CDVA of 20/20 (Fig. 1b). Figure 1c shows the changes in CDVA between preoperative and each postoperative follow-up visits. At the end of the follow-up (7 years), no eye lost more lines of CDVA, 56 eyes $(66.7 \%)$ did not change from preoperative, 20 eyes (23.81\%) gained 1 line, 4 eyes (4.76\%) gained 2 lines, and 4 eyes $(4.76 \%)$ gained more than 2 lines of CDVA. The safety index (ratio between the postoperative CDVA and the preoperative CDVA) was 1.05 throughout the 7 -year follow-up period.

\section{Predictability and stability}

Figure 2a shows a scatterplot of the attempted versus achieved spherical equivalent refraction at 1-year postsurgery. Seventy-seven eyes $(91.7 \%)$ were within $\pm 0.50 \mathrm{D}$ of the desired refraction (emmetropia) and all eyes $(100 \%)$ were within $\pm 1.00 \mathrm{D}$. The change in manifest spherical equivalent is shown in Fig. 2b. At 1-, 5- and 7years after surgery, the spherical equivalent was $-0.16 \pm$ $0.26 \mathrm{D},-0.47 \pm 0.48 \mathrm{D}$, and $-0.62 \pm 0.62 \mathrm{D}$, respectively. Multiple comparisons showed statistically significant differences among all postoperative visits (Fig. 2b; $P=$ 0.0006 ). At 5 - and 7 -years, 66.67 and $53.57 \%$ of the eyes, respectively, were within $\pm 0.50 \mathrm{D}$ of the desired refraction; while 89.29 and $80.95 \%$, respectively, were within \pm 1.00 D (Fig. 2c).

\section{Intraocular pressure, endothelial cell density, and vault}

Figure 3a shows the time course of the mean IOP over the follow-up period. A slight statistically significant reduction was found between preoperatively and 1 year after surgery $(13.02 \pm 1.77 \mathrm{mmHg}$ and $12.54 \pm 1.53$ $\mathrm{mmHg}$, respectively, $P=0.02$ ). Subsequently, the mean IOP remained stable over the 7-years of follow-up $(12.9 \pm 1.77 \mathrm{mmHg}$ and $12.69 \pm 1.64 \mathrm{mmHg}$ at 5 - and 7 years, respectively, $P=0.07$ ). Figure $3 \mathrm{~b}$ shows the changes in IOP between preoperative and each postoperative follow-up visits. At the end of the follow-up, the largest proportion of the eyes showed a reduction in IOP (37 eyes, $44.1 \%$ ), in 22 eyes (26.2\%) the IOP did not change from the preoperative value, 16 eyes $(19.0 \%)$ experienced an increased $1-2 \mathrm{mmHg}$, and 9 eyes (10.7\%) had an increased 3-4 mmHg. No significant increase in IOP (> $20 \mathrm{mmHg}$ or an increase higher than $5 \mathrm{mmHg}$ ) occurred in any case throughout the 7-year of follow-up.

Figure 4 shows the ECD over the follow-up. There were no significant changes in the mean ECD at any timepoint $(P=0.07)$. The loss in ECD from the preoperative baseline compared with the last follow-up visit was $2.6 \%$.

The mean postoperative vault was reduced from $400 \pm$ $180 \mu \mathrm{m}$ at 1 year to $355 \pm 160 \mu \mathrm{m}$ at 5 years postoperatively $(P<0.0001)$. Subsequently, it remained stable from 5 to 7 years after surgery $(348 \pm 150 \mu \mathrm{m}$ at 7 years; $P=$ 0.07 ). Figure 5 shows the postoperative distribution of vault. No eyes showed a vault higher than $800 \mu \mathrm{m}$ at any timepoint. Around $20 \%$ of eyes had a vault lower than $200 \mu \mathrm{m}$ throughout the whole follow-up.

\section{Adverse events and secondary surgeries}

There were no intraoperative complications, and no eye required ICL explantation or exchange. Over the whole 


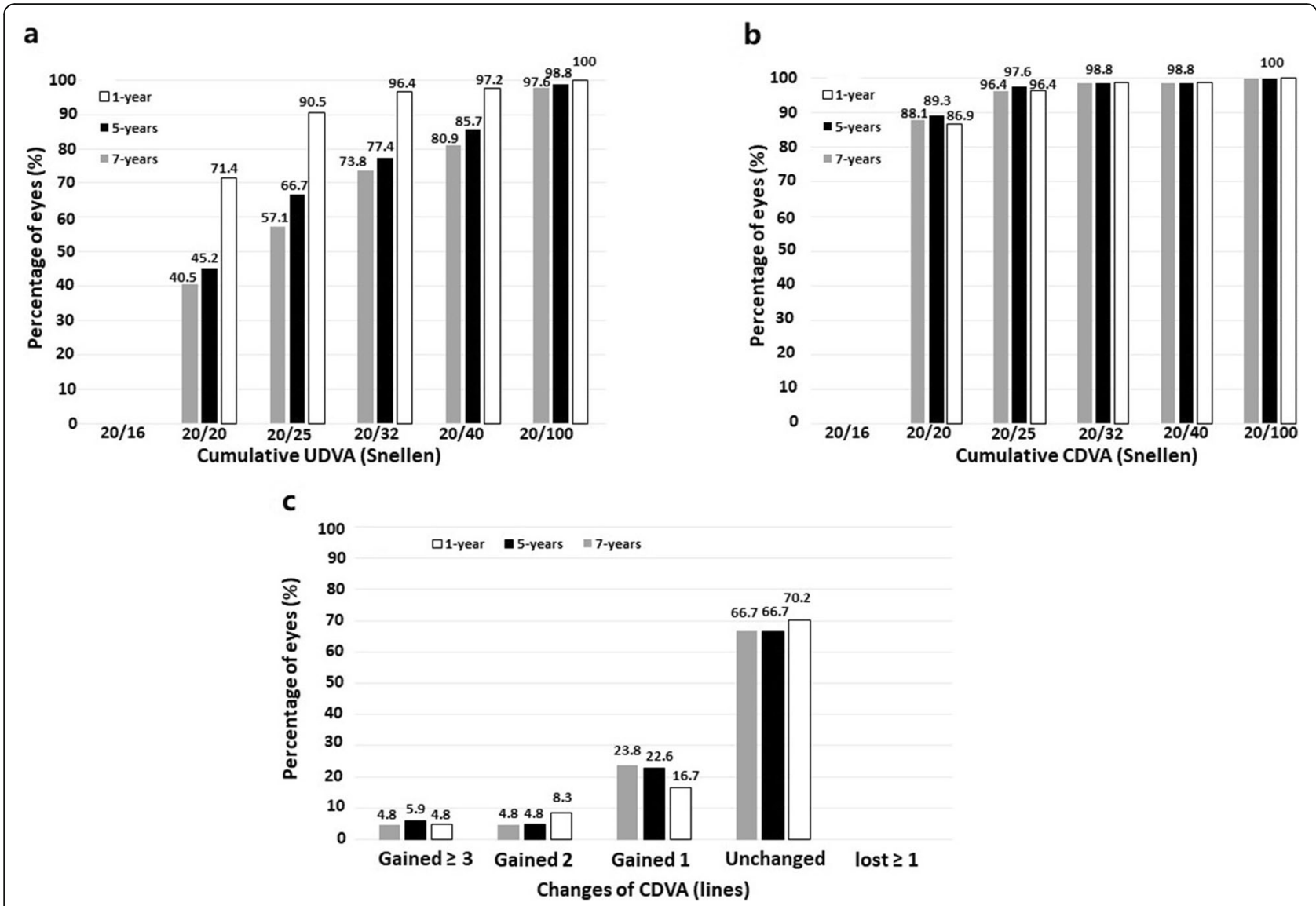

Fig. 1 Cumulative uncorrected distance visual acuity (UDVA) (a) and corrected distance visual acuity (CDVA) (b) at 1-, 5- and 7-years post-surgery. c Variation in CDVA between preoperative and each postoperative follow-up visits

follow-up period, no cases of anterior subcapsular opacity, cataract, pigment dispersion glaucoma, pupillary block, or other vision-threatening complications were reported. Two eyes of the same patient required laser touch up to correct residual refractive error 2 years after ICL implantation. In 4 eyes (4.76\%), the ICL was rotated $90^{\circ}$ to vertical orientation due to a high vault at the 1 day postoperative visit. In the four eyes, the postoperative vault dropped below $500 \mu \mathrm{m}$, and the visual and refractive outcomes were stable over the entire followup. At the end of the follow-up, the IOP change from preoperative ranged -1 to $+1 \mathrm{mmHg}$, and the ECD loss ranged between 1.9 and $2.9 \%$. In summary, the longterm results in these 4 eyes were similar to the whole group where ICL was implanted horizontally.

\section{Discussion}

The present study reports the outcomes throughout 7 years of follow-up in patients implanted with the V4c ICL model. It aimed to evaluate whether the outstanding visual and refractive results, and low adverse event rates previously reported with the V4c ICL model in shorter- follow-up studies will persist over a longer follow-up period.

During the first 5 years of follow-up, the safety index was similar to those previously reported at various postoperative intervals [1], remaining stable also up to the 7year follow-up period (1.05). At all follow-up visits, more than $95 \%$ of the eyes achieved a CDVA of $20 / 25$ or better and more than $85 \%$ a CDVA of $20 / 20$. These results support the excellent stability of the CDVA outcomes, and thus confirm the safety of the procedure. The efficacy index at 1 year was 1.01, this agrees with those previously found, that show short-term efficacy indices of 1.00 or greater. This result implies that the postoperative UDVA is equal to or better than preoperative CDVA. However, longitudinal studies show that this index worsens slightly over time $[8,9]$. The current study confirms this trend in a longer follow-up since the efficacy index at 5 years was worse than at 1 year, and in turn, at 7 years, it worsened compared to 5 years $(1.01,0.85$ and 0.80 at 1-, 5- and 7-years after surgery, respectively). It is important to note that the efficacy index is based on postoperative UDVA. Hence, it would be directly 


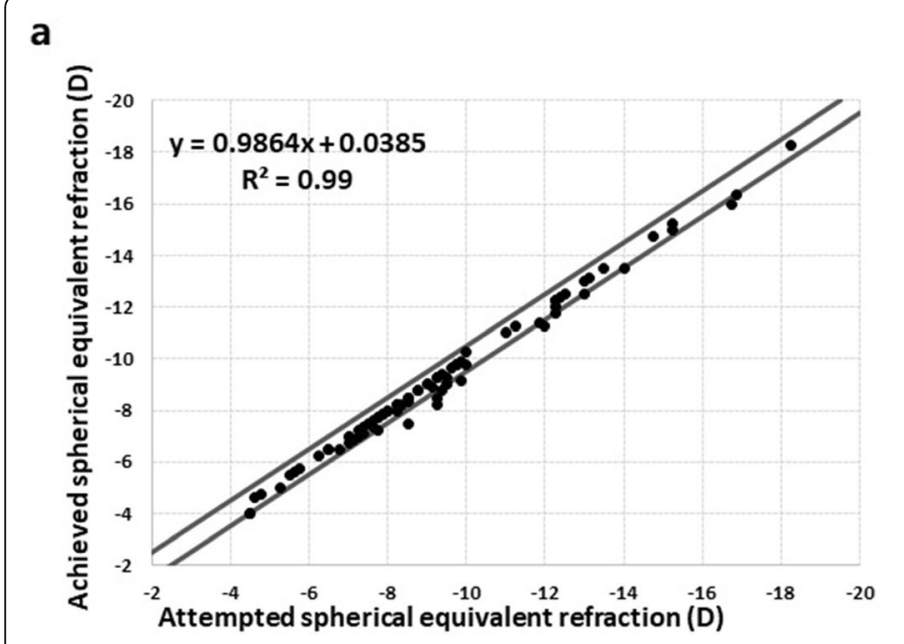

b

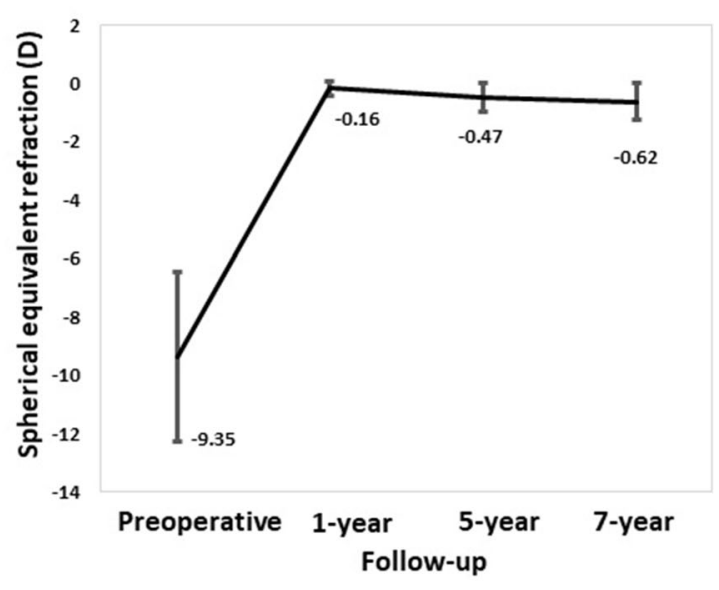

C

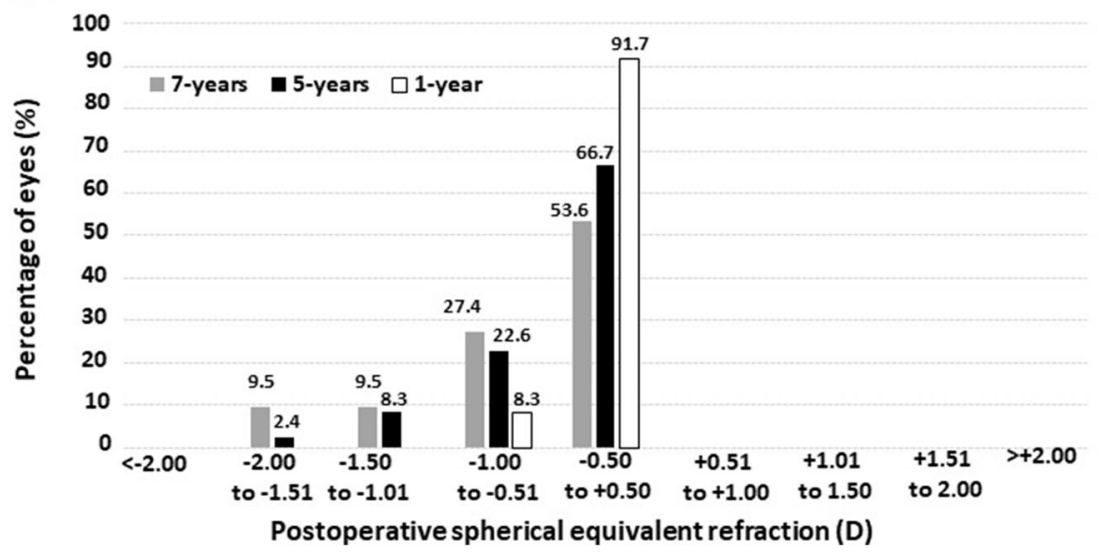

Fig. 2 Plot of attempted versus achieved spherical equivalent at 1-year post surgery (predictability) (a) Time course (b) and accuracy (c) of manifest spherical equivalent over the follow-up

affected by a change in the refractive error over time. As will be discussed below, our study showed an increase in the mean spherical equivalent of around $-0.50 \mathrm{D}$ between 1 and 7 years after surgery. Igarashi et al. [13] found the increase in axial length is the main factor for myopia progression after ICL implantation. Although the causes for this slight myopia progression could be multifactorial, the excellent outcomes of CDVA in terms of stability make it plausible to think that it was related to a continuous axial elongation and not to any other source associated with the ICL implantation procedure. Hence, this could affect any other refractive procedure.

Our refractive outcomes also confirmed satisfactory predictability results. At 1 year, 91.7 and $100 \%$ of eyes were within $\pm 0.50 \mathrm{D}$ and $\pm 1.00 \mathrm{D}$ of emmetropia, respectively. This finding agrees with those previously reported since almost all the studies reported that $100 \%$ of $\pm 1.00 \mathrm{D}$ over their postoperative periods [7-10, 22-25]. This accuracy rate slightly decreased over time [7-9]. At 5- and 7-years, 90.2 and 82.4\%, respectively, were within $\pm 1.00 \mathrm{D}$ (Fig. 2c). As previously indicated, these changes may be due to an axial elongation occurring over time. However, it should be noted that the mean spherical equivalent preoperatively was $-9.35 \mathrm{D}$ and at 7-years after surgery was $-0.62 \mathrm{D}$ while the change in the mean spherical equivalent throughout the 7 years was around -0.5 D. Hence, these values confirm the excellent refractive results of this procedure. Furthermore, for those cases that residual myopia provides an unsatisfactory UDVA level, a laser touch-up can be effectively and safely planned to correct residual refractive error after ICL implantation.

Although all previous studies had a shorter followup (up to 5 years), the satisfactory visual and refractive results previously reported were expected to remain throughout the 7 years of the follow-up period of our study. However, beyond the visual and refractive outcomes, studies spanning more than 5 years are crucial for evaluating physiological changes and potential adverse events associated with any intraocular 
a

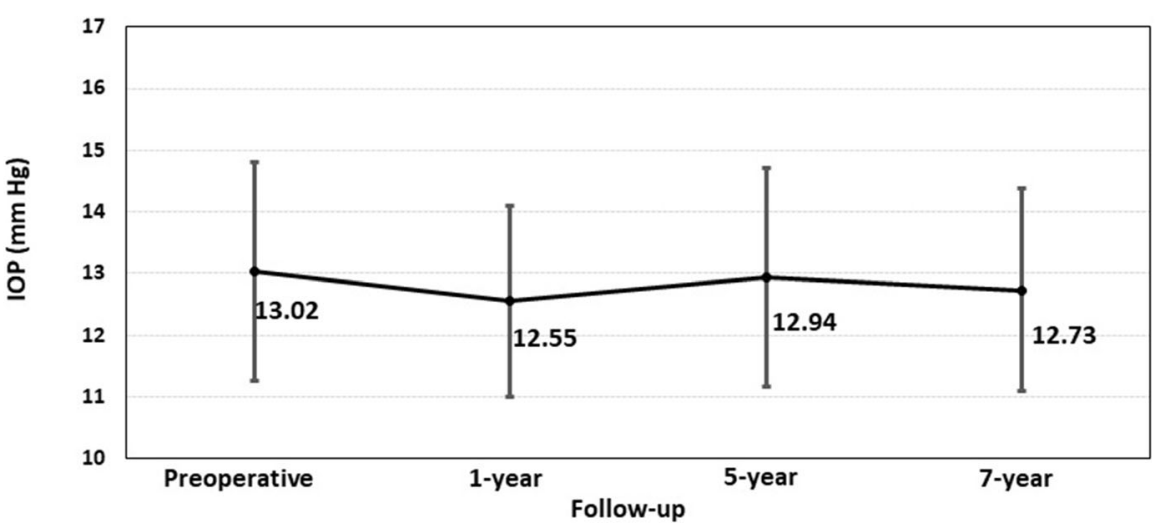

b

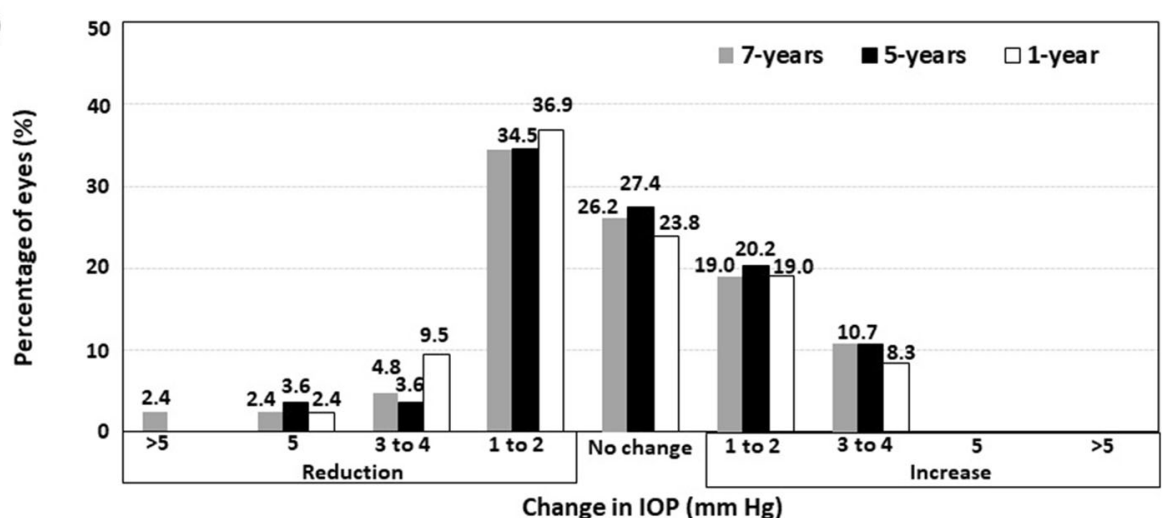

Fig. 3 The time course of the mean IOP over the whole follow-up period (a) and variation in intraocular pressure (IOP) between preoperative and each postoperative follow-up visits (b)

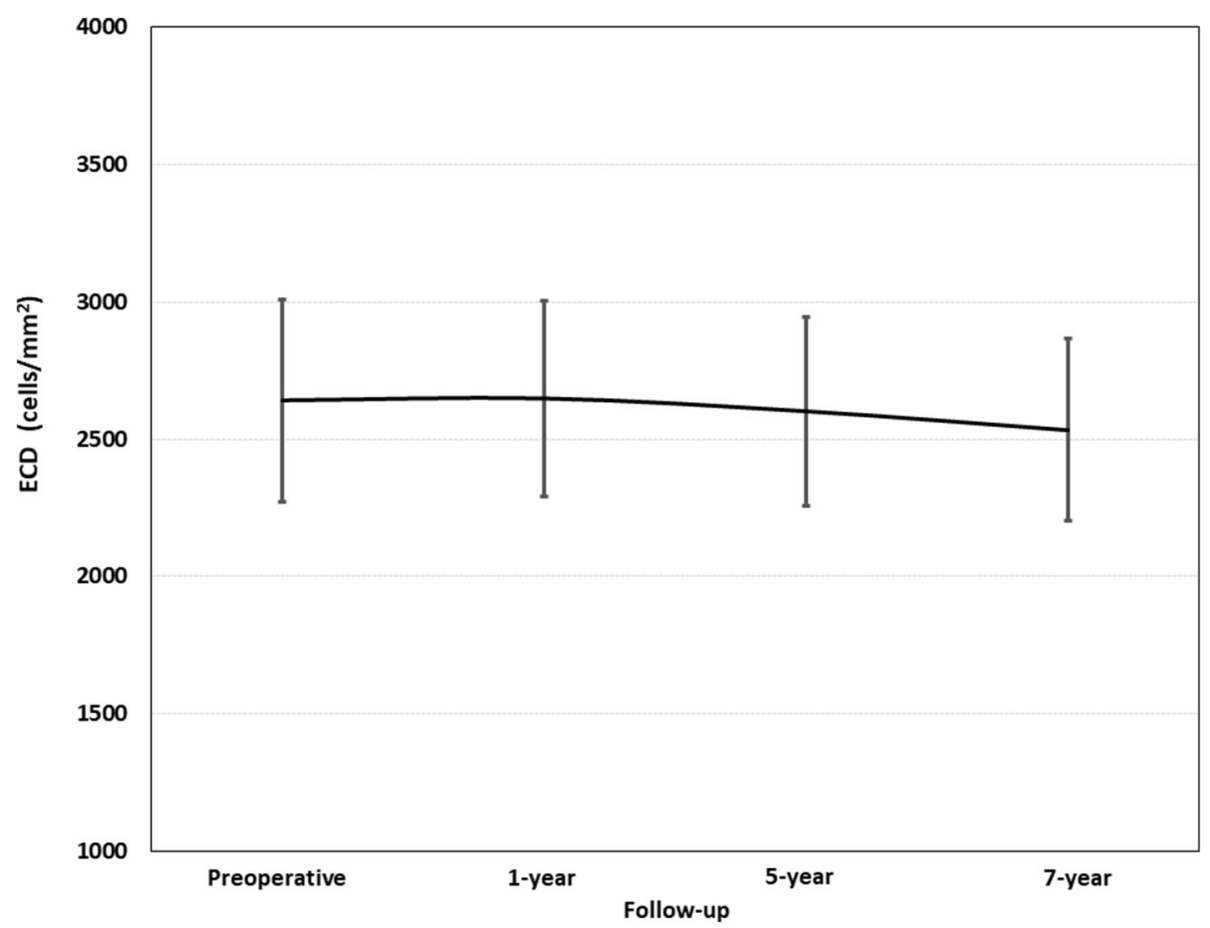

Fig. 4 Change in mean endothelial cell density (ECD) (cells $/ \mathrm{mm}^{2}$ ) throughout the entire follow-up period 


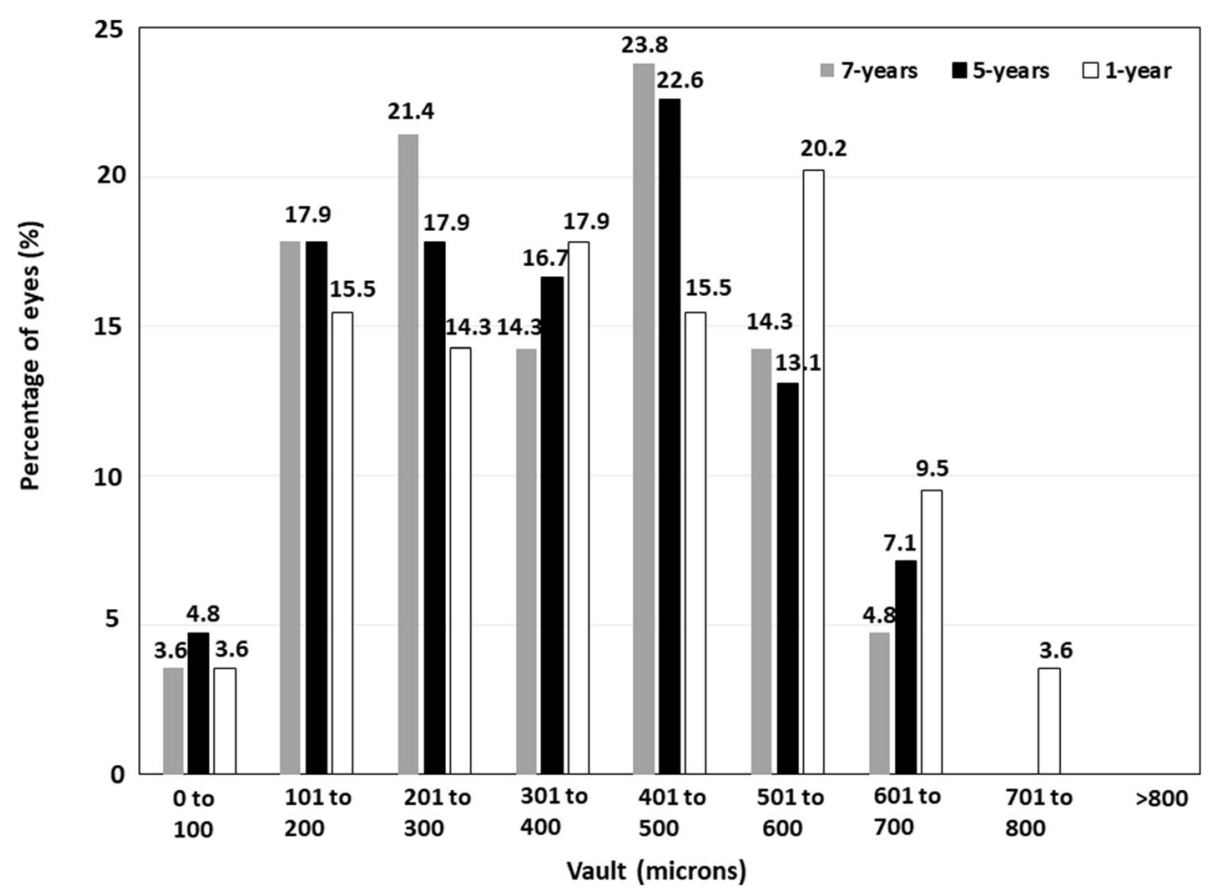

Fig. 5 Distribution of eyes according to the vault, measured in microns, at 1-, 5- and 7-years post-surgery

procedure. The rate of adverse events with the previous ICL models (such as cataracts, ECD loss, pigment dispersion syndrome) were showed to increase with time $[12,16,17,20]$.

Guber et al. [12] reported that the rate of lens opacity development increased around 15\% between 5 and 7 years after ICL V4 implantation. After 8 years of ICL V4 implantation, the incidence of cataract formation reported by Igarashi et al. [13] was higher than that reported in studies with a shorter follow-up. Choi et al. [14] showed that lens opacity developed after ICL V4 implantation occurred at a mean of $7.3 \pm 2.2$ years postoperatively. Nakamura et al. [16] reported that $10.5 \%$ of the eyes developed an anterior cataract during the 5- to 10-year follow-up period. The prevalence of cataracts after ICL V4c implantation has been reported to be around $0.17 \%$ [1], however, the longest follow-up studies published to date only reached a maximum of 5 years follow-up [6-8]. In this study, extending the follow-up to 7 years, we did not find cataract formation in any cases. One of the main risk factors associated with developing anterior capsular cataract development with the older ICL models was a low vault. Fernandes et al. [4], in a review of the potential complications of the previous models of ICL, reported that in $33.8 \%$ of ICL-induced cataracts, the vault was lower than $200 \mu \mathrm{m}$. In this study, around $20 \%$ of the eyes had a vault lower than $200 \mu \mathrm{m}$ throughout the follow-up. These findings corroborate that the central hole of the V4c ICL model prevents cataract development, even in eyes with a low vault.
Regarding IOP, the central hole offers surgical advantages over non-hole ICL models since no preoperative iridotomy or intraoperative iridectomy is necessary to prevent IOP increase related to pupillary block or chronic pigment dispersion [4]. Shimizu et al. [7] showed comparative IOP values between eyes implanted with a hole-equipped ICL and a non-hole ICL, over a follow-up period of 5 years. Furthermore, previous studies with short-, medium- and long-term follow-up showed that there no significant variation of IOP over time [1] after ICL V4c implantation. However, it is important to be cautious because the longest follow-up to date was 5 years. In the Guber et al. [12] study, around $13 \%$ of the cases developed ocular hypertension at a mean of 7.3 years after ICL V4 implantation. In our study, the mean IOP remained stable over the 7-years of follow-up (Fig. 3a). At 7 years of the surgery, the largest proportion of the eyes showed a reduction in IOP from the preoperative value, and no significant increase in IOP (> $20 \mathrm{mmHg}$ or an increase higher than $5 \mathrm{mmHg}$ ) occurred in any case throughout the 7-year of follow-up (Fig. 3b). These results suggest that central hole prevent the IOP increase that may be associated with pupillary block or chronic pigment dispersion [4]. In addition to the central hole ICL design, it should be noted that our study showed no eyes with a vault higher than $800 \mu \mathrm{m}$ at any timepoint. To prevent a postoperative high vault, we rotated the ICL $90^{\circ}$ to vertical orientation in four eyes so that the distance between the ICL and the ATA was higher than $800 \mu \mathrm{m}$ in the postoperative visit of the first 
day. In all 4 eyes, the postoperative vault dropped below $500 \mu \mathrm{m}$ after ICL vertical rotation, all the parameters studied were stable over the entire follow-up, and no associated complications were found in these four eyes. The sulcus has a vertically oval shape, with the vertical diameter longer than the horizontal one [26, 27]. Consequently, it is expected that the ICL rotation to a vertical alignment should reduce the vault, and thus the postoperative complications related to a high vault, without the need of an ICL exchange for a smaller size one. Of note, before performing this surgical manoeuvre, it is mandatory to confirm that the vertical diameter is longer than the horizontal one. Currently, intraoperative OCT allows us to measure the vault intraoperatively, hence, for those cases with an extreme intraoperative vault, the ICL can be rotated during the same surgical session. The intraoperative OCT represent an outstanding tool to prevent potential complications related to an inadequate vault. It would be interesting to carry out future studies to establish intraoperative vault values safety cut-off.

Finally, our results did not reveal a statistically significant change in ECD over the 7 years of follow-up. The loss in ECD from the preoperative baseline compared to the last follow-up visit was $2.6 \%$. This is in good agreement with those previously reported, suggesting that the ICL does not induce a significant ECD loss over long periods [1]. However, it is interesting to note that Yang et al. [10] found that excessively high vault values increased the risk of ECD loss. Hence, the vertical ICL rotation manoeuvre performed in 4 eyes $(4.76 \%)$ may have avoided a risk factor (high vault) for a potential ECD loss over time.

\section{Conclusion}

The outcomes of the present study indicate that the use of the V4c ICL model for the correction of myopia was overall satisfactory in terms of effectiveness, safety and stability during 7-years of follow-up, which shows its long-term viability as a surgical option for the correction of myopia.

\section{Abbreviations \\ ICL: Implantable Collamer Lens; UDVA: Uncorrected distance visual acuity; CDVA: Uncorrected distance visual acuity; IOP: Intraocular pressure; ECD: Endothelial cell density; ACD: Anterior chamber depth; TIA: Trabecular- iris angle; CLD: Crystalline lens rise; OCT: Optical coherence tomography; WTW: White-to-white; ATA: Angle-to-angle diameter}

\section{Acknowledgements}

Not applicable.

\section{Authors' contributions}

LFVC: Conception and design of the study; Analysis and interpretation of data; Writing the manuscript. BA: Conception and design of the study; Analysis and interpretation of data; Critical revision of the manuscript. $\mathrm{CL}$ : Conception and design of the study; Analysis and interpretation of data; Critical revision of the manuscript. DMC: Conception and design of the study; Analysis and interpretation of data; Critical revision of the manuscript; Supervision. JFA: Conception and design of the study; Analysis and interpretation of data; Critical revision of the manuscript; Supervision. All authors read and approved the final manuscript.

\section{Funding}

This study was supported in part by an unrestricted grant from STAAR Surgical to the Fernández-Vega Ophthalmological Institute.

\section{Availability of data and materials}

Not applicable

\section{Declarations}

\section{Ethics approval and consent to participate}

This study was conducted in compliance with the tenets of the Declaration of Helsinki, and full ethical approval from the Fernández-Vega

Ophthalmological Institute was obtained. After receiving a complete description of the nature of the study and the possible consequences of surgery, all patients provided informed consent.

\section{Consent for publication}

Not applicable

\section{Competing interests}

The authors declare that they have no competing interests.

\section{Author details}

${ }^{1}$ Fernández-Vega Ophthalmological Institute, Avda. Dres. Fernández-Vega 114, 33012 Oviedo, Spain. ${ }^{2}$ Optometry and Vision Department, Faculty of Optics and Optometry, University Complutense de Madrid, Madrid, Spain.

Received: 22 February 2021 Accepted: 22 May 2021

Published online: 11 June 2021

\section{References}

1. Montés-Micó R, Ruiz-Mesa R, Rodríguez-Prats JL, Tañá-Rivero P. Posteriorchamber phakic implantable collamer lenses with a central port: a review. Acta Ophthalmol. 2021;99(3):e288-301.

2. Fernández-Vigo Jl, Macarro-Merino A, Fernández-Francos J, De-PabloGómez-de-Liaño L, Martínez-de-la-Casa JM, García-Feijóo J, et al. Computational study of aqueous humor dynamics assessing the vault and the pupil diameter in two posterior-chamber phakic lenses. Invest Ophthalmol Vis Sci. 2016;57(11):4625-31.

3. Kawamorita T, Shimizu K, Shoji N. Effect of hole size on fluid dynamics of a posterior-chamber phakic intraocular lens with a central perforation by using computational fluid dynamics. Graefes Arch Clin Exp Ophthalmol. 2016;254(4):739-44.

4. Fernandes P, González-Méijome JM, Madrid-Costa D, Ferrer-Blasco T, Jorge J, Montés-Micó R. Implantable collamer posterior chamber intraocular lenses: a review of potential complications. J Refract Surg. 2011;27(10):765-76.

5. Packer M. Meta-analysis and review: effectiveness, safety, and central port design of the intraocular collamer lens. Clin Ophthalmol. 2016;10:1059-77.

6. Gonzalez-Lopez F, Bouza-Miguens C, Tejerina V, Mompean B, OrtegaUsobiaga J, Bilbao-Calabuig R, et al. Long-term assessment of crystalline lens transparency in eyes implanted with a central-hole phakic collamer lens developing low postoperative vault. J Cataract Refract Surg. 2021;47(2): 204-10.

7. Shimizu K, Kamiya K, Igarashi A, Kobashi H. Long-term comparison of posterior chamber phakic intraocular lens with and without a central hole (hole ICL and conventional ICL) implantation for moderate to high myopia and myopic astigmatism: consort-compliant article. Medicine (Baltimore). 2016;95(14):e3270

8. Alfonso JF, Fernández-Vega-Cueto L, Alfonso-Bartolozzi B, Montés-Micó R, Fernández-Vega L. Five-year follow-up of correction of myopia: posterior chamber phakic intraocular lens with a central port design. J Refract Surg. 2019;35(3):169-76.

9. Fernández-Vega-Cueto L, Lisa C, Esteve-Taboada JJ, Montés-Micó R, Alfonso JF. Implantable collamer lens with central hole: 3 -year follow-up. Clin Ophthalmol. 2018;12:2015-29. 
10. Yang W, Zhao J, Sun L, Zhao J, Niu L, Wang X, et al. Four-year observation of the changes in corneal endothelium cell density and the correlated factors after implantable collamer lens V4C implantation. Br J Ophthalmol. 2021;105(5):625-30.

11. Yang W, Zhao J, Zhao J, Shen Y, Niu L, A R, et al. Changes in anterior lens density after Implantable Collamer Lens V4C implantation: a 4-year prospective observational study. Acta Ophthalmol. 2021;99(3):326-33.

12. Guber I, Mouvet V, Bergin C, Perritaz S, Othenin-Girard P, Majo F. Clinical outcomes and cataract formation rates in eyes 10 years after posterior phakic lens implantation for myopia. JAMA Ophthalmol. 2016;134(5):487-94.

13. Igarashi A, Shimizu K, Kamiya K. Eight-year follow-up of posterior chamber phakic intraocular lens implantation for moderate to high myopia. Am J Ophthalmol. 2014;157(3):532-9.e1.

14. Choi JH, Lim DH, Nam SW, Yang CM, Chung ES, Chung TY. Ten-year clinical outcomes after implantation of a posterior chamber phakic intraocular lens for myopia. J Cataract Refract Surg. 2019;45(11):1555-61.

15. Moya T, Javaloy J, Montés-Micó R, Beltrán J, Muñoz G, Montalbán R. Implantable Collamer Lens for myopia: assessment 12 years after implantation. J Refract Surg. 2015;31(8):548-56.

16. Nakamura T, Isogai N, Kojima T, Yoshida Y, Sugiyama Y. Posterior chamber phakic intraocular lens implantation for the correction of myopia and myopic astigmatism: a retrospective 10-year follow-up study. Am J Ophthalmol. 2019;206:1-10.

17. Sanders DR. Anterior subcapsular opacities and cataracts 5 years after surgery in the Visian Implantable Collamer Lens FDA trial. J Refract Surg. 2008;24(6):566-70

18. Lindland A, Heger H, Kugelberg M, Zetterström C. Vaulting of myopic and toric Implantable Collamer Lenses during accommodation measured with Visante optical coherence tomography. Ophthalmology. 2010;117(6):124550.

19. Schmidinger G, Lackner B, Pieh S, Skorpik C. Long-term changes in posterior chamber phakic intraocular collamer lens vaulting in myopic patients. Ophthalmology. 2010;117(8):1506-11.

20. Gonvers M, Bornet C, Othenin-Girard P. Implantable contact lens for moderate to high myopia: relationship of vaulting to cataract formation. J Cataract Refract Surg. 2003;29(5):918-24.

21. Alfonso JF, Lisa C, Fernández-Vega Cueto L, Belda-Salmerón L, Madrid-Costa D, Montés-Micó R. Clinical outcomes after implantation of a posterior chamber collagen copolymer phakic intraocular lens with a central hole for myopic correction. J Cataract Refract Surg. 2013;39(6):915-21.

22. Kamiya K, Shimizu K, Igarashi A, Kitazawa Y, Kojima T, Nakamura T, et al. Posterior chamber phakic intraocular lens implantation: comparative, multicentre study in 351 eyes with low-to-moderate or high myopia. Br J Ophthalmol. 2018;102(2):177-81.

23. Garcia-de la Rosa G, Olivo-Payne A, Serna-Ojeda JC, Salazar-Ramos MS, Lichtinger A, Gomez-Bastar A, et al. Anterior segment optical coherence tomography angle and vault analysis after toric and non-toric Implantable Collamer Lens V4C implantation in patients with high myopia. Br J Ophthalmol. 2018;102(4):544-8.

24. Lisa C, Naveiras M, Alfonso-Bartolozzi B, Belda-Salmerón L, Montés-Micó R, Alfonso JF. Posterior chamber collagen copolymer phakic intraocular lens with a central hole to correct myopia: one-year follow-up. J Cataract Refract Surg. 2015;41(6):1153-9.

25. Niu L, Miao H, Tian M, Fu D, Wang X, Zhou X. One-year visual outcomes and optical quality of femtosecond laser small incision lenticule extraction and Visian Implantable Collamer Lens (ICL V4c) implantation for high myopia. Acta Ophthalmol. 2020;98(6):e662-7.

26. Oh J, Shin HH, Kim JH, Kim HM, Song JS. Direct measurement of the ciliary sulcus diameter by 35-megahertz ultrasound biomicroscopy. Ophthalmology. 2007;114(9):1685-8.

27. Biermann J, Bredow L, Boehringer D, Reinhard T. Evaluation of ciliary sulcus diameter using ultrasound biomicroscopy in emmetropic eyes and myopic eyes. J Cataract Refract Surg. 2011;37(9):1686-93.

Ready to submit your research? Choose BMC and benefit from:

- fast, convenient online submission

- thorough peer review by experienced researchers in your field

- rapid publication on acceptance

- support for research data, including large and complex data types

- gold Open Access which fosters wider collaboration and increased citations

- maximum visibility for your research: over $100 \mathrm{M}$ website views per year

At BMC, research is always in progress.

Learn more biomedcentral.com/submissions 